\title{
Gestione nefrologica della lista d'attesa per trapianto nei primi otto anni di attività di un singolo Centro Trapianti
}

\author{
Paolo De Paolis, Roberto Colonnelli, Antonio Favarò, Fatma Salem, Benedetta Bartoli, Salvatore Di Giulio \\ UOC Nefrologia-Dialisi e Trapianto. AO S. Camillo-Forlanini, Roma
}

\begin{abstract}
Management of kidney transplantation waiting list: eight years experience
Potential recipients pre-transplantation screening represents an essential step of kidney transplantation. Screening is mainly directed to identify conditions before transplantation and treating underlying diseases of potential recipients. Therefore, it's crucial to identify the patients at higher risk after transplantation and those who are not suitable for transplantation. A waiting period can modify the basal clinical picture necessitating a systematic clinical reassessment to confirm transplantation suitability. Our aim was to evaluate a nephrological management of waiting list recipients in a single Transplant Center in the first eight years of activity. We report our evaluation, the interaction with a network of specialists and the modification of our clinical behaviour considering the increase of high risk transplantation candidates, especially because of cardiovascular and infectious comorbidities and the consequent relationship with patients' Nephrology Units. Our experience shows that a periodical and systematic re-evaluation allows to extend patients' transplant program suitability with better outcomes after transplantation.
\end{abstract}

Keywords: High risk candidates, MACE, Pre-transplantation screening programs, Waiting list

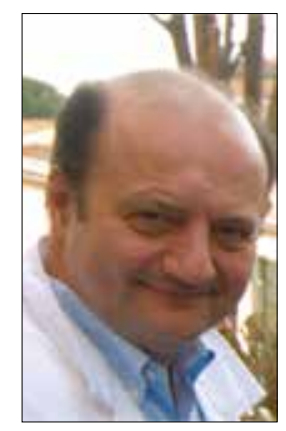

Paolo De Paolis

\section{Introduzione}

Viene qui riportata l'esperienza di avvio e di gestione della lista d'attesa per trapianto dal Maggio 2007 al Maggio 2015 del nostro Centro Trapianti "POIT", Polo Ospedaliero Interaziendale Trapianti di Roma, nato dalla collaborazione tra l'AO S. Camillo-Forlanini e I'INMI L. Spallanzani per le attività di trapianto di rene e rene-pancreas.

Vengono descritte le numerose problematiche cliniche che sono presenti frequentemente nei potenziali candidati al trapianto, con particolare attenzione all'attività nefrologica che viene svolta curando e garantendo una continua assi-

Accepted: October 30, 2015

Published online: February 29, 2016

Indirizzo per la corrispondenza:

Paolo De Paolis

UOS Nefrologia e Dialisi in Aree Critiche e Trapianto

AO S. Camillo-Forlanini

Circonvallazione Gianicolense 87

00152 Roma

pdepaolis@scamilloforlanini.rm.it stenza, durante tutta la permanenza in lista d'attesa, in stretta collaborazione con altre figure specialistiche della nostra AO e con le Nefrologie di provenienza dei pazienti.

\section{Metodi}

L'attività iniziale di valutazione per l'idoneità al trapianto è cominciata diversi mesi prima dell'avvio dell'attività chirurgica e riguardava principalmente i pazienti, seguiti dalla nostra UO Nefrologia e Dialisi, già in studio o precedentemente iscritti presso altri Centri Trapianti regionali o extraregionali. Con l'avvio dell'attività di trapianto, abbiamo, poi, osservato un aumento di richieste di valutazione e inserimento in lista da altre Nefrologie e/o Centri Dialisi laziali e/o extraregionali e abbiamo offerto la possibilità di eseguire anche gli esami strumentali basali e obbligatori a quei pazienti che non avevano potuto eseguirli presso la propria Nefrologia di provenienza.

All'inizio dell'attività, la maggior parte dei pazienti valutati presentava un basso profilo di rischio complessivo e un'età anagrafica contenuta. In seguito, però, l'aumento del numero di richieste ha prodotto l'emergere di una serie di problematiche cliniche, spesso non risolte dalle stesse Nefrologie di provenienza. Ciò ci ha indotto ad attivare dei percorsi specialistici interaziendali per poter affrontare tali problematiche nel minor tempo possibile. L'avvio di un programma di doppio trapianto renale 
da donatore marginale ha comportato il progressivo aumento delle richieste di pazienti con età sempre più avanzata, che ci ha obbligato a creare ulteriori percorsi di studio in risposta alle situazioni complesse e articolate che questi pazienti presentano.

In particolare, sono stati attivati percorsi specialistici di secondo livello per le seguenti problematiche cliniche:

- problematiche cardio-vascolari per pazienti con patologia cardiaca ischemica e/o con scompenso cardiaco di grado medio e con valvulopatie e/o disturbi del ritmo;

- problematiche infettivologiche, con particolare attenzione alla ricerca di forme di TBC latente. Ciò è dovuto al significativo numero di pazienti extracomunitari afferenti al nostro Centro (81 su 637 , pari al $12.7 \%$ degli iscritti in lista, provenienti soprattutto dall'est europeo, dal sud-est asiatico, dall'area maghrebina e, in minor misura, dall'Africa centrale e dall'America meridionale). Oltre alla TBC, abbiamo problematiche di epatopatia HCV-correlata, con replicazione attiva e, quindi, con frequente necessità di biopsia epatica e di terapia protratta sino al raggiungimento di una risposta virologica sostenuta. Dal 2013, inoltre, dopo l'autorizzazione, rilasciataci dal Centro Nazionale Trapianti (CNT), al programma di trapianto per i pazienti portatori di infezione da HIV, è stata avviata una valutazione ad hoc comprendente un'attenta valutazione degli effetti collaterali, soprattutto cardiologici e metabolici, della terapia HAART (Highly Active Antiretroviral Therapy) a cui questi pazienti sono esposti per il controllo della replicazione virale;

- problematiche immunologiche nei pazienti iperimmuni per pregressi trapianti alle quali consegue spesso la necessità di espiantare l'organo ancora in sede;

- problematiche legate alla valutazione dei potenziali candidati al doppio trapianto che per l'età l'avanzata necessitano di un apposito percorso di studio vascolare e cardiologico;

- problematiche legate alle numerose comorbidità dei pazienti diabetici, per i quali sia posta indicazione a trapianto combinato di rene-pancreas 0 , in assenza di nefropatia, di pancreas isolato;

- problematiche chirurgiche con necessità di intervento prima dell'inserimento in lista o durante il mantenimento nella stessa, per nefrectomia monolaterale in pazienti con APKD, reflusso vescico-ureterale severo, colelitiasi, iperparatiroidismo, neoplasie renali e così via.

Come da indicazione del Centro Regionale Trapianti Lazio (CRTL) e del CNT, a conclusione della valutazione multi specialistica, si calcola per ciascun paziente, in base alle problematiche presenti, il case mix ricevente, diviso in standard, lieve, intermedio o elevato (1). Un rischio complessivo eccessivamente elevato è causa di non idoneità al trapianto, che viene immediatamente comunicata e formalizzata direttamente al paziente e alla Nefrologia di provenienza. Se, viceversa, la valutazione evidenzia singoli fattori di rischio inferiori, questi vengono co- municati prima al paziente, attraverso la consegna del certificato di iscrizione che viene sottoscritto dall'interessato e, successivamente, sia alla Nefrologia di provenienza che al CRTL.

Nella Tabella I viene riportato il profilo delle diverse comorbidità per la quantificazione del rischio complessivo del CNT.

Periodicamente, inoltre, è eseguita una rivalutazione delle condizioni dei pazienti. Per i pazienti che presentano un rischio basso (standard o lieve), essa avviene almeno una volta all'anno, mentre, per quelli che presentano un rischio maggiore (intermedio o elevato), si programma una valutazione multi specialistica ogni sei mesi presso il nostro Centro.

In parallelo all'attività clinica, viene sempre garantito l'aggiornamento della cartella clinica elettronica regionale (LURTO), con la quale il CRTL individua il potenziale candidato per l'allocazione degli organi per ciascun potenziale donatore. La cartella elettronica comprende dati anamnestici, immunolo-

TABELLA I - Elenco della quantificazione delle comorbidità per il calcolo del case mix del ricevente

\begin{tabular}{|c|c|c|}
\hline \multirow[t]{12}{*}{ Cardiomiopatia } & Assente & 0 \\
\hline & Ischemia lieve & 1 \\
\hline & Ischemia moderata & 2 \\
\hline & con IMA non complicato (>6 mesi) & 3 \\
\hline & Valvolare (grado lieve) & 1 \\
\hline & Valvolare (grado medio) & 2 \\
\hline & Dilatativa (FE tra 50-60\%) & 1 \\
\hline & Dilatativa (FE tra 40-50\%) & 2 \\
\hline & Dilatativa (FE tra 35-40\%) & 3 \\
\hline & Ipertensiva (con danno d'organo) & 3 \\
\hline & Ipertensiva (grado lieve-medio) & 1 \\
\hline & $\begin{array}{l}\text { Aritmia stabile } \\
\text { (F.A.-pace maker permanente) }\end{array}$ & 2 \\
\hline \multirow[t]{4}{*}{ Cerebrovascolare } & Assente & 0 \\
\hline & TIA pregresso & 1 \\
\hline & RIND pregresso & 2 \\
\hline & Ictus non invalidante, più episodi TIA & 3 \\
\hline \multirow[t]{4}{*}{ Vasculopatia periferica } & assente & 0 \\
\hline & $\begin{array}{l}\text { calcificazioni estese } \\
\text { (non sintomatologia) }\end{array}$ & 1 \\
\hline & Ischemia periferica con sintomi & 3 \\
\hline & $\begin{array}{l}\text { con Pat Aneurismatica o stenosi } \\
\text { carotidea }\end{array}$ & 3 \\
\hline \multirow[t]{3}{*}{ Broncopneumopatia } & Assente & 0 \\
\hline & con I.R. lieve (compreso ex fumatori) & 1 \\
\hline & $\begin{array}{l}\text { con I.R. di grado medio (o attuale } \\
\text { fumatore) }\end{array}$ & 2 \\
\hline \multirow[t]{5}{*}{ Infezione HBV-HCV } & Assente & 0 \\
\hline & Presente (DNA/RNA -, Alt normali) & 1 \\
\hline & $\begin{array}{l}\text { Presente (DNA/RNA +, Alt normali) } \\
\text { Presente (DNA/RNA + Alt }\end{array}$ & 2 \\
\hline & $\begin{array}{l}\text { Presente (DINA/RINA +, Alt } \\
\text { aumentate) }\end{array}$ & 3 \\
\hline & con Epatite cronica attiva & 3 \\
\hline $\begin{array}{l}\text { Neoplasia } \\
\text { (non cutanee) }\end{array}$ & Assenti & 0 \\
\hline
\end{tabular}


TABELLA I - Continua

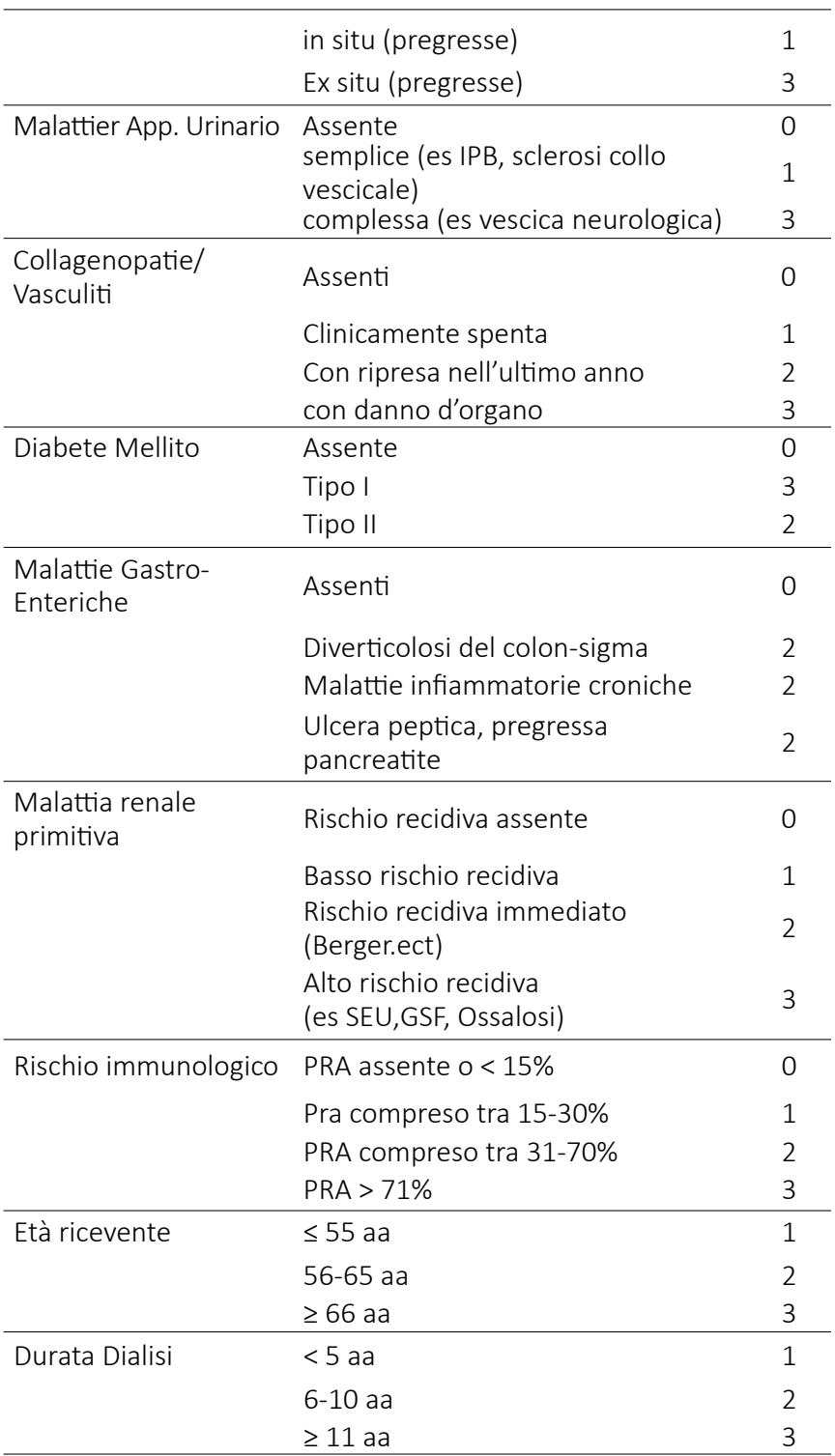

gici e infettivologici e i risultati di analisi biochimiche e di esami strumentali effettuati nel tempo per ogni paziente inserito in lista. Al momento dell'offerta dell'organo per il potenziale candidato, il nefrologo del Centro dispone, così, di una sintesi clinica per confermare celermente l'idoneità dello stesso al trapianto.

Altro aspetto da sempre particolarmente curato in conformità alle direttive della Regione Lazio (2) è quello di garantire la massima comunicazione durante le fasi di valutazione tra la Nefrologia di provenienza e il Centro Trapianti. Ovviamente, le nefrologie di provenienza sono coinvolte anche nel momento della possibile allocazione dell'organo, per ottenere la conoscenza della reale situazione clinica del ricevente al momento del trapianto.

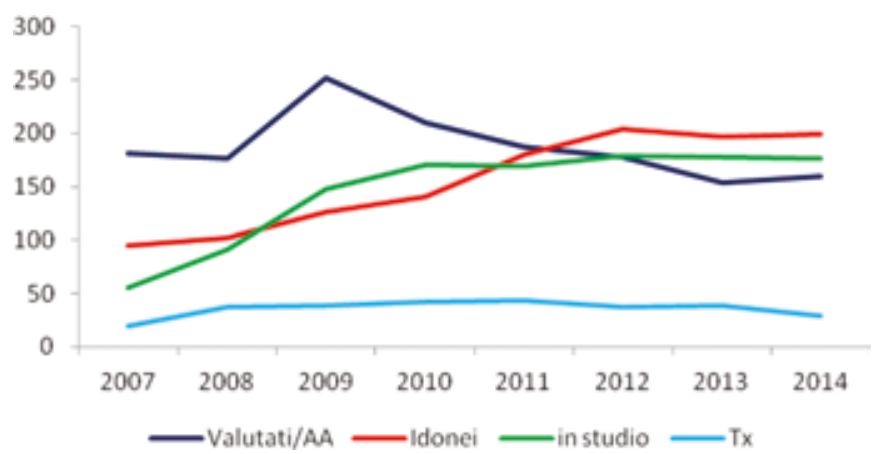

Fig. 1 - Profilo dell'attività al 31 Dicembre 2014.

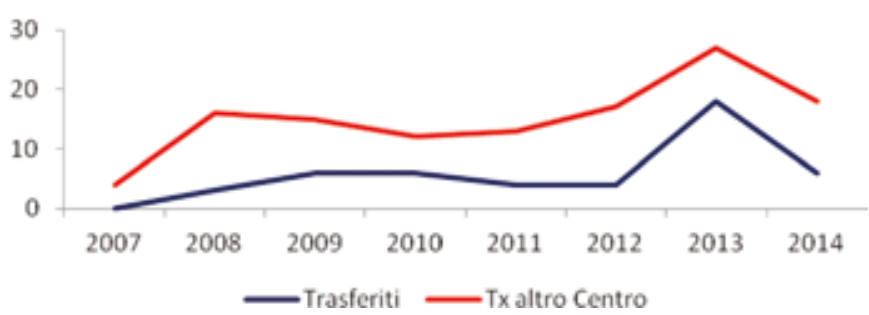

Fig. 2 - Numero di pazienti iscritti presso il POIT e trasferiti presso altri Centri Trapianti regionali o trapiantati presso Centri extraregionali al 31 Dicembre 2014

\section{Risultati e considerazioni}

Negli otto anni di riferimento (2007-2014), si sono rivolti al nostro Centro Trapianti 1471 pazienti. Di questi, 637 (43.3\%) sono stati ritenuti idonei e inseriti in lista. Nella Figura 1 viene riportato in valore assoluto al 31 Dicembre di ciascun anno il numero dei pazienti studiati, di quelli ritenuti idonei e inseriti in lista, di quelli ancora in studio e di quelli trapiantati presso il nostro Centro. L'osservazione ha rilevato che, nei primi tre anni di attività, abbiamo ricevuto numerose richieste di valutazione di candidati che, in passato, erano stati ritenuti non idonei da altri Centri Trapianti regionali. Negli anni successivi, invece, nonostante una flessione generale del numero di richiedenti una valutazione di idoneità, abbiamo osservato un aumento graduale del numero di pazienti ritenuti idonei e inseriti in lista.

Nella Figura 2 viene riportato il numero di pazienti iscritti presso il nostro Centro che, nel periodo di osservazione, hanno chiesto il trasferimento presso un altro Centro Trapianti regionale e di quelli trapiantati presso un secondo Centro Trapianti extraregionale.

Nella Figura 3 viene riportato il numero di valutazioni di secondo livello eseguite nel periodo di osservazione dagli specialisti referenti del nostro Centro Trapianti per le problematiche cliniche più frequenti, che sono state: cardiologiche, con necessità di ecocardiografia con stress farmacologico o scintigrafia miocardica basale e sotto sforzo in regime di Day Hospital o di coronarografia in regime di ricovero ordinario, vascolari, con l'esecuzione di un'angio-TC in regime ambulatoriale o con esami arteriografici in regime di ricovero ordi- 


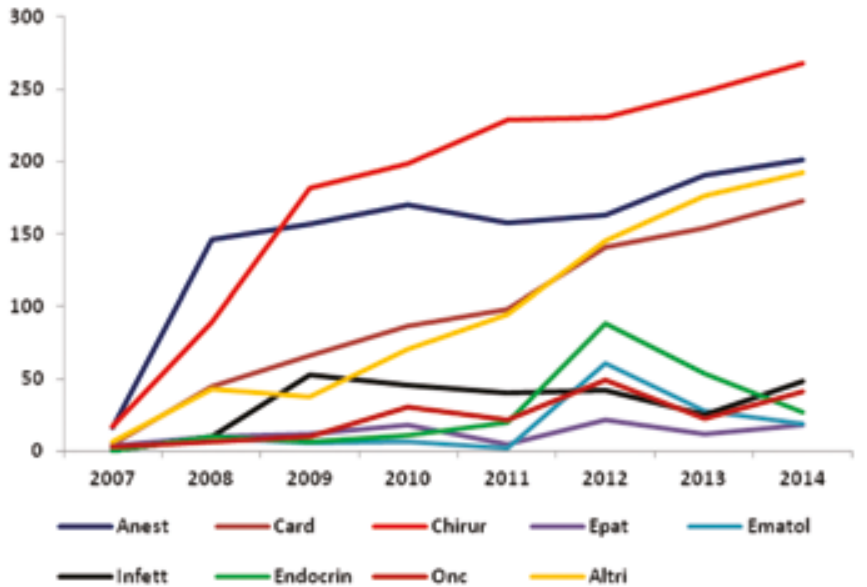

Fig. 3 - Numero di valutazioni di secondo livello per specialità al 31 Dicembre 2014

nario, e oncologiche, con follow up condivisi con gli oncologi, volti al raggiungimento di un periodo congruo di negatività del quadro clinico e a ottenere il minimo valore di rischio di recidiva dopo trapianto. Le valutazioni testimoniano anche il volume di attività del nefrologo del Centro Trapianti nel raccordare tutti gli specialisti coinvolti e la stretta collaborazione che è stata avviata con numerose branche specialistiche della nostra AO.

Di seguito vengono riportati i risultati ottenuti avviando $i$ pazienti a percorsi specialistici di secondo livello.

\section{Problematiche cardiache}

Su un totale di 195 potenziali candidati con problematiche cardiologiche, sono state eseguite 78 coronarografie in altrettanti pazienti, che hanno portato a 64 casi in PTCA con posizionamento di stent, in 5 in intervento chirurgico di BPAC (By Pass Aorto Coronarico); 9 sono risultate negative. In totale, 60 pazienti sono stati ritenuti idonei. Di questi, 23 sono stati trapiantati, 5 sono ancora in lista d'attesa e 32 sono stati esclusi per ulteriori eventi clinici maggiori. Un paziente in emodialisi è in attesa di trapianto combinato di rene-cuore per scompenso cardiaco congestizio.

\section{Problematiche infettivologiche legate alla TBC}

La problematica della TBC latente ha prodotto, dopo l'approfondimento con esami colturali e valutazioni infettivologiche ripetute, l'avvio a una terapia profilattica con Isoniazide pre-trapianto per 9 mesi in 60 pazienti di cui 21 sono ancora in lista, 35 sono stati trapiantati e 4 sono stati esclusi definitivamente per altre cause.

\section{Problematiche HCV-correlate}

Sono stati valutati e avviati a terapia con Peg-Interferon e Ribavirina 21 pazienti. Di questi, 5 sono ancora in lista, 7 sono stati trapiantati (di cui 1 HCVRNA positivo trapiantato da donatore HCV positivo), 3 sono risultati non responder e 6 sono stati esclusi per complicanze intercorrenti. Ovviamente, con la recente possibilità di utilizzare nuovi farmaci antivirali, la strategia terapeutica si sta modificando, ma, per il momento, tali farmaci non sono stati ancora autorizzati per i pazienti in emodialisi nella Regione Lazio.

\section{Problematiche legate all'infezione da HIV}

Sono stati valutati 11 pazienti di cui 2 , al momento, in lista attiva, 3 in fase di studio e 6 esclusi definitivamente per inidoneità clinica.

\section{Problematiche in pazienti con pregresso trapianto}

Sono stati inseriti in lista 96 pazienti con pregresso trapianto $(15.1 \%$, valore sovrapponibile a quanto riportato dall'USRDS del 2014) (3), di cui 3 al terzo trapianto. In 80 di questi pazienti (83.3\%), è stato eseguito l'espianto del rene (per cause settiche, reflusso vescico-ureterale o cause immunologiche) prima del completamento della valutazione per I'inserimento in lista, in modo da avere almeno 6 mesi di osservazione sierologica prima dell'iscrizione in lista. Di questi 96 pazienti, al momento 37 sono ancora in lista, 33 sono stati trapiantati presso il nostro Centro e 7 presso Centri extraregionali, 6 sono stati trasferiti presso un altro Centro, 7 sono stati esclusi definitivamente per complicanze sopraggiunte e 6 sono deceduti, per cause non legate all'espianto, durante il periodo di mantenimento in lista.

\section{Problematiche per potenziali riceventi di rene da donatore marginale}

In totale, sono stati inseriti in lista 60 pazienti per ricevere un doppio trapianto di rene. Le indicazioni all'inserimento in lista per doppio trapianto sono state l'età maggiore di 60 anni, l'assenza di problematiche vascolari e il consenso sottoscritto del paziente. Al momento, 6 pazienti sono ancora in lista d'attesa, 36 sono stati trapiantati e 18 sono stati esclusi per sopraggiunta inidoneità clinica.

\section{Problematiche in pazienti nefropatici diabetici}

Negli anni sono stati valutati 40 pazienti. Di questi, 15 hanno ricevuto un trapianto combinato, mentre 1 ha ricevuto un trapianto di pancreas isolato. Sono ancora in attesa 4 candidati per il combinato e 2 per il pancreas isolato, 3 , anche se ritenuti idonei, sono stati esclusi per complicanze cardio-vascolari, 4 sono in fase di studio e 11 sono stati ritenuti non idonei al trapianto combinato.

Considerando, invece, la complessità clinica generale dei pazienti inseriti in lista, stratificata, secondo il cosiddetto case 


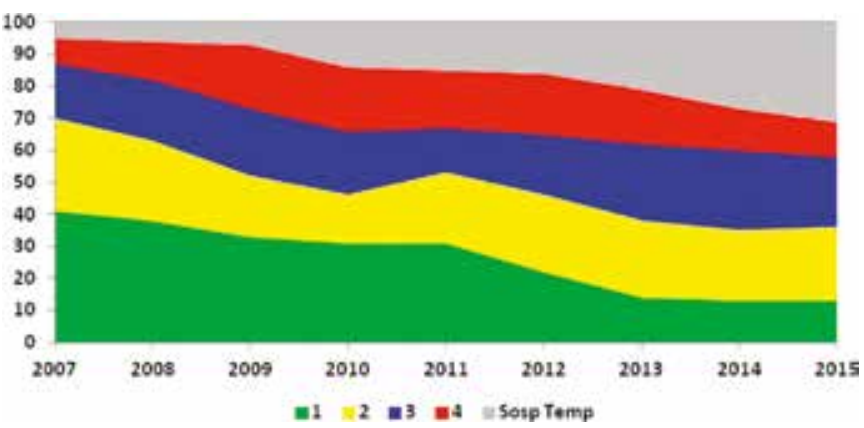

Fig. 4 - Modificazioni in percentuale nel tempo delle classi di case mix e dei sospesi temporaneamente al Maggio 2015 (1: rischio standard, 2: rischio lieve, 3: rischio intermedio, 4: rischio elevato).

mix ricevente del CNT, in rischio standard, lieve, intermedio e elevato, si riportano, nella Figura 4, le modificazioni in percentuale delle diverse classi nel tempo, comprese quelle sospese temporaneamente. Da sottolineare che, nel 2009, primo anno in cui osservammo un significativo aumento dei pazienti iscritti a rischio elevato (26\%), i tempi per arrivare all'iscrizione (riportati come $\mathrm{M}+/$-DS e range) nei 4 gruppi di rischio sono stati rispettivamente: standard $3.9 \pm 2.5$, range $1-13$ mesi, lieve $5.3 \pm 4.5$, range $1-15$ mesi, intermedio $9.4 \pm 7.8$, range $5-27$ mesi, ed elevato $13.4 \pm 11.5$, range 6-39 mesi. Viceversa, da notare che, nel Maggio 2015, grazie ai percorsi multi specialistici avviati nel 2010, i tempi per raggiungere l'iscrizione sono stati rispettivamente: standard $3.2 \pm 2.3$, range 1-12 mesi, lieve $5.7 \pm 4.3$, range $1-23$ mesi, intermedio $8.1 \pm 6$, range $1-31$ mesi, ed elevato $10.3 \pm 9.2$, range 3-35 mesi.

Valutando i tempi di studio, considerati dalla prima visita eseguita presso il nostro Centro Trapianti al momento dell'iscrizione in lista, il nostro intervento è servito a ridurli nei gruppi a maggior rischio, mentre non ha inciso sui gruppi a rischio inferiore. II tempo necessario per completare la valutazione rimane un problema aperto nella nostra realtà regionale, come confermato dai recenti dati del Registro Dialisi e Trapianti della Regione Lazio nel report 2013-2014. In tale report, sono mostrate le percentuali di pazienti in valutazione che passano dal 20.4\% del totale dei pazienti nel 2013 al 22\% nel 2014 (4).

Nella Figura 5 vengono riportate, al Maggio 2015, le percentuali delle diverse classi di rischio dei pazienti sospesi temporaneamente. La maggior parte dei pazienti all'atto dell' inserimento in lista risultava a rischio intermedio-elevato (86.2\% dei sospesi totali). Nella Figura 6 viene riportata l'attività complessiva di trapianto svolta fino al Maggio 2015.

Nella Figura 7 viene riportata, invece, la suddivisione rispetto al case mix della popolazione dei pazienti trapiantati e di quelli ancora in lista al Maggio 2015, dove risulta che, nel nostro Centro, il case mix elevato non pregiudica la possibilità di ricevere un trapianto. La maggiore complessità clinica ha comportato, nella nostra popolazione, un aumento del tempo medio per arrivare al trapianto. Infatti, nei primi anni di attività, il tempo di attesa pre-trapianto della nostra popolazione

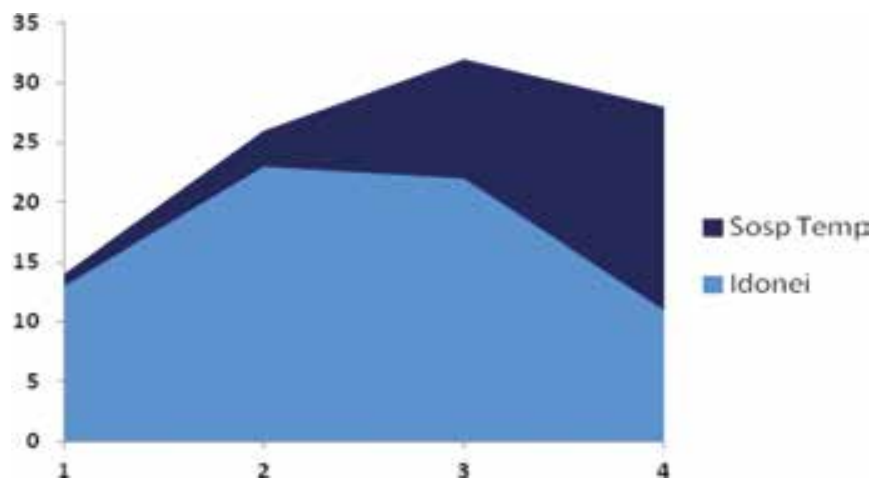

Fig. 5 - Percentuali degli idonei e dei sospesi, separati per classe di case mix al Maggio 2015 (1: rischio standard, 2: rischio lieve, 3: rischio intermedio, 4: rischio elevato).

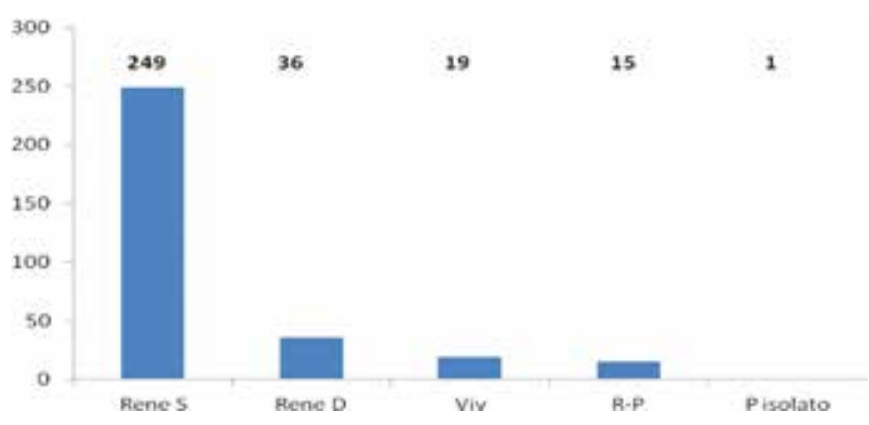

Fig. 6 - Attività complessiva di trapianto al Maggio 2015.

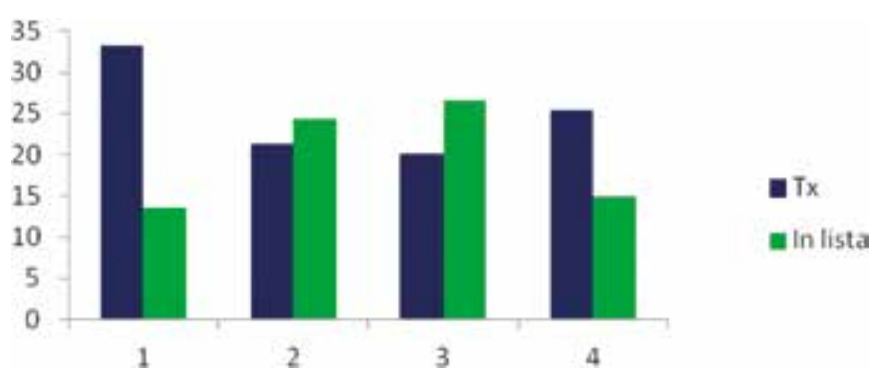

Fig. 7 - Percentuale dei trapiantati e dei pazienti in lista attiva separati per case mix al 31 Maggio 2015 (1: rischio standard, 2: rischio lieve, 3: rischio intermedio, 4: rischio elevato).

era inferiore a quello riportato dal CNT nel report dell'attività del 2011 (5) (rispettivamente POIT 19 vs 27 mesi CNT), mentre, successivamente, nel report dell'attività del 2014 (6), la nostra popolazione che riceveva il trapianto si è assestata su medie sovrapponibili a quelle del CNT (nel 2014 rispettivamente POIT 27 mesi vs 26 mesi CNT). La bassa percentuale dei pazienti in attesa di trapianto a rischio elevato, invece, era dovuta alla necessità di sospendere temporaneamente molti di questi pazienti per sopraggiunte problematiche cliniche (Fig. 5). II motivo è riportato nella Figura 8, dove risulta che, durante l'osservazione nel periodo in lista, per il sopraggiungere di un peggioramento delle condizioni cliniche, i pazienti venivano temporaneamente sospesi e ristudiati; 


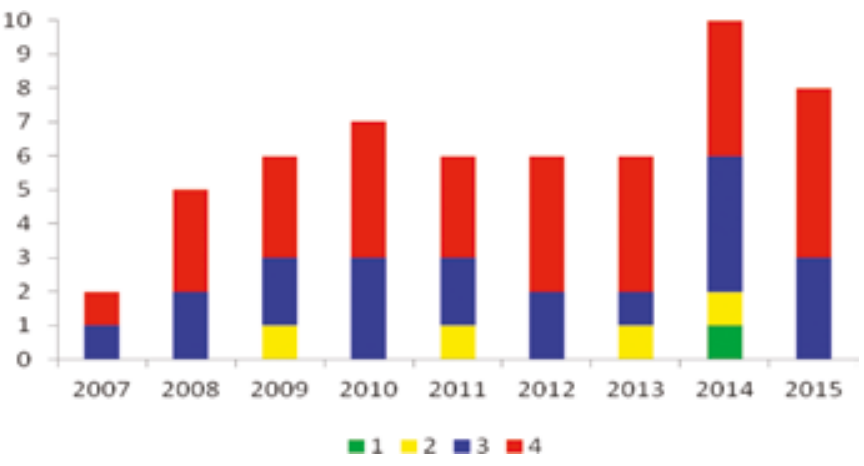

Fig. 8 - Numero di pazienti iscritti e, poi, sospesi definitivamente, separati per case mix al Maggio 2015 (1: rischio standard, 2: rischio lieve, 3: rischio intermedio, 4: rischio elevato).

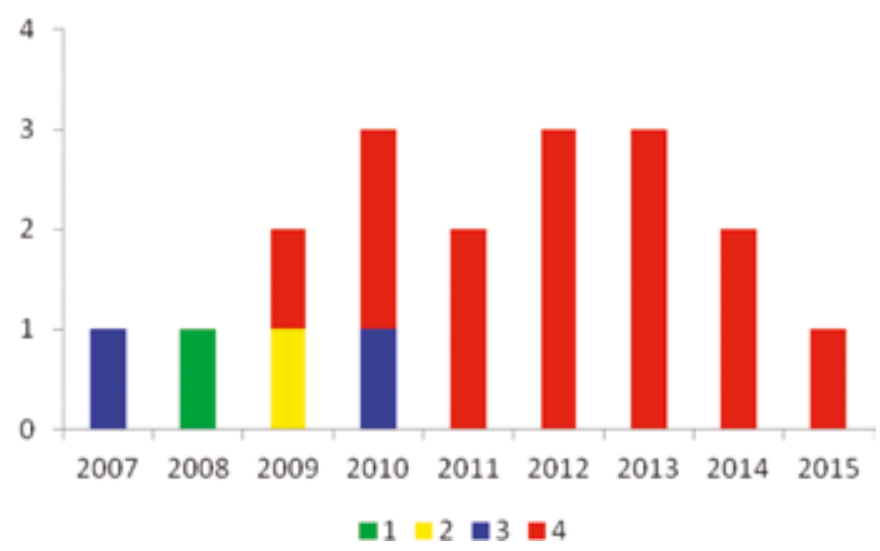

Fig. 9 - Numero di decessi di pazienti in lista, separati per case mix al Maggio 2015 (1: rischio standard, 2: rischio lieve, 3: rischio intermedio, 4: rischio elevato).

se veniva confermato il peggioramento clinico, erano esclusi definitivamente dalla lista.

La maggior parte delle esclusioni riguardava, ovviamente, pazienti a rischio intermedio ed elevato.

Nella Figura 9 viene riportato il numero dei decessi osservati durante la permanenza in lista. Il tasso di mortalità risulta essere significativamente più basso rispetto ai dati del CNT, che riporta un tasso di mortalità/annua dell'1.8\% (Fig. 10). In entrambe le Figures 8 e 9, i pazienti vengono suddivisi per classe di rischio all'inserimento in lista ed emerge che sia nel caso dei decessi che nel caso dei pazienti esclusi definitivamente, la percentuale maggiore appartiene alla classe di rischio più elevata, che presentava anche un'età media +/- DS più elevata (rispettivamente $58 \pm 12$ aa, $60 \pm 11$ aa), segno di una complessità clinica maggiore e di una maggiore suscettibilità a eventi clinici più gravi.

II numero dei candidati valutati e la periodicità dei controlli giustificano il volume di attività che viene maggiormente svolta in regime ambulatoriale e di ricovero in Day Hospital ed è riportato nella Figura 11. Si nota come, nel tempo, mentre il numero di valutazioni ambulatoriali risulta in lieve aumento,

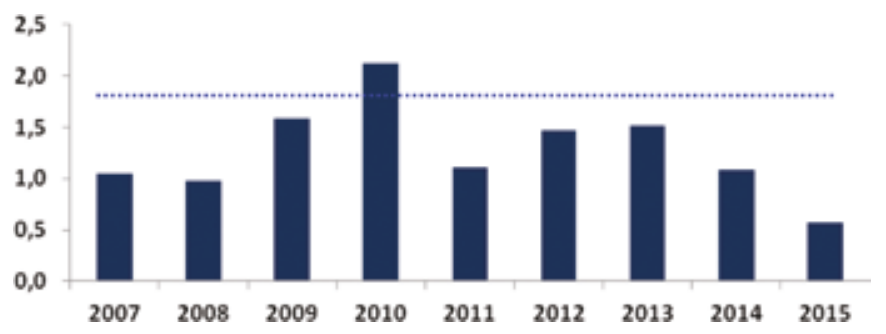

Fig. 10 - Tasso di mortalità/aa del POIT vs CNT (1.8\%/aa).

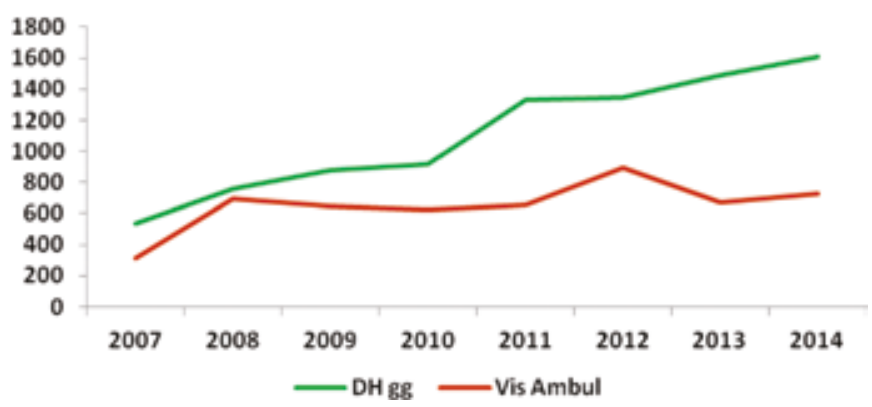

Fig. 11 - Attività ambulatoriale e di DH nel tempo.

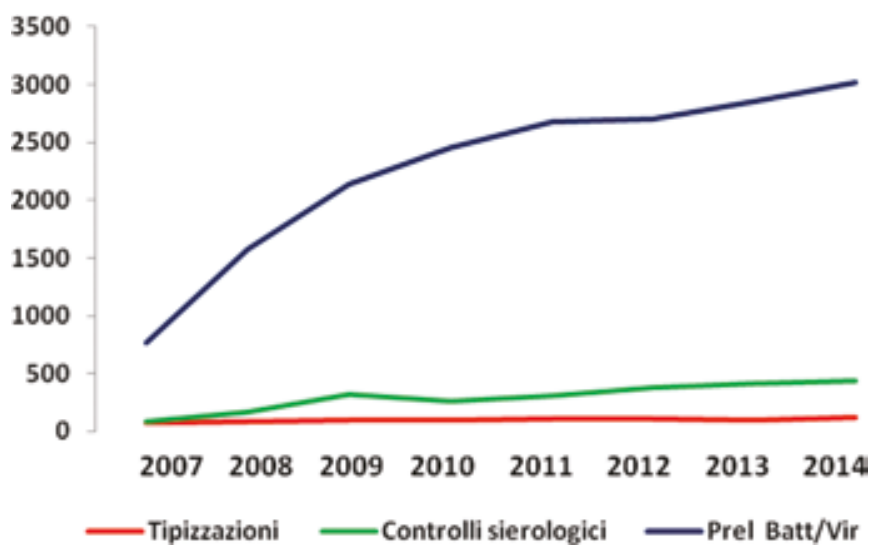

Fig. 12 - Numero prelievi eseguiti nel tempo.

la complessità clinica dei potenziali candidati da iscrivere o di quelli già iscritti in lista e da ricontrollare nel tempo ha richiesto un maggior numero di approfondimenti multi specialistici, soprattutto di pertinenza cardio-vascolare, da eseguire necessariamente in regime di $\mathrm{DH}$ con un significativo aumento dell'attività. Nella Figura 12, invece, si riporta il numero dei prelievi eseguiti per lo studio e il monitoraggio immunologico e infettivologico, effettuati negli anni su tutta la popolazione in osservazione. Data la specificità degli esami e l'alta percentuale di pazienti (113 su 637: 17.7\%) con problematiche infettivologiche (HBV-HCV-HIV-TBC o altre comorbidità legate all'alto numero di pazienti extracomunitari) se ne preferisce l'esecuzione nella nostra AO. Da sottolineare che tali controlli infettivologici vengono rieseguiti nei pazienti già iscritti, al ri- 
torno da periodi di permanenza temporanea nei loro paesi di origine in base alle diverse endemie potenzialmente presenti.

La gestione e la conoscenza approfondita dello stato clinico dei pazienti nel periodo d'attesa rappresentano una seria difficoltà per un Centro Trapianti. Tale stato, infatti, richiede uno stretto controllo e, soprattutto, una collaborazione con le Nefrologie di provenienza, che, nella nostra esperienza, si sono sempre dimostrate pronte e rapide nel comunicare eventi significativi che potessero modificare l'idoneità clinica dei propri pazienti.

Nel corso degli anni, la possibilità di offrire programmi peculiari come quelli del doppio trapianto di rene, il combinato rene-pancreas e programmi in pazienti con HCV o HIV ha prodotto l'arrivo di una significativa popolazione con numerose comorbidità e la necessità di offrire una valutazione estesa e multi specialistica. Per questo motivo, abbiamo identificato singoli referenti per le diverse branche di competenza con appositi percorsi diagnostico-terapeutici multi specialistici all'interno del nostro $\mathrm{DH}$, utilizzando tutte le risorse della nostra AO. Tale strategia ha l'obiettivo di raggiungere un'uniformità di valutazione nel tempo e di creare una condivisione allargata delle scelte cliniche adottate.

Da notare, inoltre, come il significativo numero di pazienti a rischio medio-elevato giustifichi l'aumento delle percentuali di quelli sospesi temporaneamente, vista la loro predisposizione a sviluppare complicanze più serie. Al Maggio 2015, infatti, la percentuale dei sospesi risulta aumentata al 31\% della popolazione iscritta in lista, da un valore, dopo il primo anno di attività, del 5\%. La nostra esperienza si avvicina ai dati dell'USRDS (3) in cui il $57 \%$ dei pazienti iscritti in lista è in fase attiva mentre il $43 \%$ rimane in una fase temporaneamente inattiva. Questo ha comportato parallelamente un maggior carico di lavoro per la nostra Nefrologia, che ha dovuto riverificare l'idoneità al trapianto dei pazienti, prendendoli in carico.

Viceversa, la percentuale dei pazienti a basso rischio, che risultano essere più frequentemente giovani e in migliori condizioni cliniche, è andata a diminuire. Ciò è da imputare al fatto che costoro sono stati trapiantati sia presso il nostro Centro che da altri Centri extraregionali, opportunità, quest'ultima, ridotta per i pazienti con rischio basale elevato che, con più difficoltà, vengono inseriti in Centri Trapianti fuori regione.

Con la possibilità di utilizzare donatori marginali, la nostra esperienza ha mostrato che i 99 donatori utilizzati (35.1\% del numero totale dei donatori), di età in media \pm DS di $69 \pm 7$ anni, hanno permesso l'allocazione di reni (trapianto singolo o doppio) in altrettanti riceventi di $65 \pm 6$ anni in tempi più brevi. Infatti, il tempo d'attesa per arrivare al trapianto nella nostra popolazione generale è di $27.3 \pm 6.5$ mesi, mentre, con I'utilizzo di donatori marginali, i riceventi di trapianto singolo arrivano al trapianto in $17.2 \pm 16.8$ mesi, mentre i riceventi di doppio trapianto arrivano al trapianto in tempi ancora più brevi (9.6 \pm 8.27 mesi).

Infine, da notare che abbiamo ricevuto poche richieste di trasferimento dei pazienti in lista $(n=47 ; 7.3 \%)$ verso altri cen- tri. Interpretiamo questo dato come segno di apprezzamento da parte della maggioranza dei pazienti per la nostra meticolosità di studio e per il nostro monitoraggio nel tempo particolari.

\section{Conclusioni}

II candidato al trapianto richiede un'approfondita valutazione clinica multi specialistica, che inizia nelle Nefrologie di provenienza e che finisce nel Centro Trapianti. La possibilità di utilizzare donatori di età più avanzata per riceventi più anziani e il prolungato periodo di mantenimento in lista espongono tali pazienti in generale e, soprattutto, quelli a case mix aumentato a una maggiore incidenza di complicanze che ne pregiudica spesso temporaneamente, ma, a volte, definitivamente l'idoneità al trapianto. Uno stretto monitoraggio nel nostro Centro Trapianti permette una definizione sufficientemente precisa delle condizioni cliniche del candidato al trapianto, anche grazie alla stretta collaborazione con le Nefrologie di provenienza. Tale strategia proattiva è ancora possibile con il personale a disposizione grazie alle dimensioni contenute della nostra lista d'attesa e all'alta percentuale (81\%) di provenienza regionale dei nostri pazienti e garantisce l'allocazione dell'organo a un candidato in buone condizioni cliniche con risvolti positivi sull'outcome del ricevente nel post-trapianto. Per ottenere questi buoni risultati, è stato determinante l'impiego di personale medico e infermieristico esclusivamente dedicato al programma di trapianto con la presa in carico del candidato al trapianto sin dalle prime fasi della valutazione, passando per l'intervento chirurgico e, poi, seguendolo nel follow up nel tempo. In tutto questo è coinvolto non solo il personale dedicato della UOC di Nefrologia-Dialisi e Trapianto ma anche il personale scelto di altre branche specialistiche della nostra AO, soprattutto chirurghi e anestesisti e, last but not least, il personale delle Nefrologie di provenienza. Rimane prioritaria la necessità di ridurre i tempi per arrivare alla valutazione di idoneità o meno del candidato al trapianto ma, in questo, è necessario un maggiore coinvolgimento di tutta la rete sanitaria regionale.

\section{Disclosures}

Financial support: No financial support was received for this submission.

Conflict of interest: The authors have no conflict of interest.

\section{Bibliografia}

1. Centro Nazionale Trapianti. Determinazione del case mix ricevente. $2^{\text {a }}$ versione del 30 Settembre 2002

2. Regione Lazio. Percorso assistenziale al trapianto di rene. DGR n. 403 del 30 Maggio 2008.

3. United States Renal Data System. Report 2014.

4. Registro Regionale Dialisi e Trapianti del Lazio. Dipartimento di Epidemiologia del Servizio Sanitario Regionale. Regione Lazio, 2 Dicembre 2015.

5. Report 2011 del Centro Nazionale Trapianti. Attività di donazione e trapianti in Italia.

6. Report 2014 del Centro Nazionale Trapianti. Attività di donazione e trapianti in Italia. 